\title{
Durian species recognition system based on global shape representations and k-nearest neighbors
}

\begin{abstract}
Many fruit recognition systems today are designed to classify different type of fruits but there is no content-based fruit recognition system focuses on durian species. Durian, known as the king of tropical fruits, have few similar characteristics between different species where the skin have almost the same color from green to yellowish brown with slightly different shape of thorns and it is hard to differentiate them with the current methods. Sometimes it is even hard for general consumers to differentiate durian species by themselves. This work aims to contribute to an automatic content-based durian species recognition that will be able to assist users in differentiating various species of durian. Few global contour-based and region-based shape descriptors such as area, perimeter, and circularity are computed as feature vectors and K-Nearest Neighbors algorithm is used to classify the durian based on the extracted features. 10 -fold cross-validation is used to evaluate the proposed system. Experimental results have shown that the proposed feature extraction method for the durian species recognition system has successfully obtained a positive recognition rate of $100 \%$.
\end{abstract}

Keyword: Durian; Content-based; Shape feature; Kï nearest neighbors; Recognition rate; 10fold cross validation 